\title{
Conhecimento e prática das mulheres atendidas na unidade de saúde da família sobre o Papanicolaou
}

\author{
Knowledge and practice on Pap smear tests by women assisted at family health units \\ Conocimiento y práctica de las mujeres atendidas en la unidad de salud de la familia sobre \\ el Papanicolaou
}

Thatiany Rodrigues Santiago'; Magna Santos Andrade ${ }^{I I}$; Gilvânia Patrícia do Nascimento Paixão ${ }^{I I I}$

\begin{abstract}
RESUMO: O objetivo do estudo foi descrever o conhecimento e a prática sobre o Papanicolaou das mulheres entre 25 a 59 anos atendidas pela Estratégia de Saúde da Família. Trata-se de um estudo de corte transversal. Foram entrevistadas, em seus domicílios, 47 mulheres, a partir da aplicação de formulário entre os meses de agosto a setembro de 2012, residentes na área de abrangência da Unidade de Saúde da Família Nossa Senhora de Fátima, Senhor do Bonfim-BA. Os dados foram analisados através da aplicação da estatística descritiva. Os resultados demonstram que ainda existem mulheres que não realizam o Papanicolaou regularmente e, principalmente, desconhecem a finalidade do procedimento. Conclui-se que as mulheres necessitam de esclarecimento sobre o exame e seu objetivo, sendo necessária a estruturação de atividades educativas permanentes nas unidades de saúde que sensibilizem as usuárias sobre a importância da realização regular do Papanicolaou. Palavras-Chave: Neoplasias do colo do útero; esfregaço vaginal; controle do câncer; prevenção primária.
\end{abstract}

\begin{abstract}
This study aims at describing the knowledge and practice on the Pap smear tests by women between 25 and 59 years assisted at the Family Health Strategy. This is a cross-sectional study. Forty-seven (47) women residing within the area serviced by the Health Unit Family Our Lady of Fatima, Our Lord of Bonfim, Bahia, Brazil were interviewed at home on the basis of application of forms, from August to September, 2012. Data were analyzed on the basis of descriptive statistics. Results show that there are still women who do not receive regular Pap and are mostly unaware of the purpose of the procedure. Conclusions show that women need clarification about the exam and its goal, requiring permanent structure of educational activities in health facilities to sensitize women about the importance of regular Pap smears.

Keywords: Uterine cervical neoplasms; vaginal smear; knowledge; primary prevention.

RESUMEN: El objetivo de este estudio fue describir el conocimiento y la práctica sobre el Papanicolaou de mujeres 25 y 59 años atendidas por la Estrategia de Salud de la Familia. Se trata de un estudio transversal. Fueron entrevistadas en su casas 47 mujeres, desde un formulario, entre los meses de agosto a septiembre de 2012, residentes en el área de influencia de la Unidad de Salud Familiar Nuestra Señora de Fátima, Nuestra Señor de Bonfim, Bahia - Brasil. Los datos fueron analizados mediante la aplicación de estadística descriptiva. Los resultados muestran que todavía hay mujeres que no hacen Papanicolaou regular y sobre todo desconocen de la finalidad del procedimiento. Se concluye que las mujeres necesitan de aclaraciones sobre el examen y su finalidad, que requieren actividades educativas permanentes en los establecimientos de salud para sensibilizar a las mujeres acerca de la importancia de las pruebas regulares de Papanicolaou.

Palabras Clave: Neoplasias del cuello uterino; frotis vaginal; control de cáncer; prevención primaria.
\end{abstract}

\section{INTRODUÇÃO}

O câncer do colo do útero (CCU) é um grave problema de saúde pública devido às altas taxas de morbimortalidade, com incidência cerca de duas vezes maior em países em desenvolvimento se comparado aos indicadores dos países desenvolvidos ${ }^{1}$.

No Brasil estima-se uma ocorrência anual aproximadamente 18.000 casos novos de câncer cérvico-uterino, com risco de 18 casos a cada $100 \mathrm{mil}$ mulheres, sendo a terceira neoplasia mais frequente na população feminina².
O Papanicolaou é o principal exame para o diagnóstico precoce da neoplasia cérvico-uterina, sendo um método de baixo custo, fácil execução e alta eficácia para a detecção de alterações cervicais, com ampla utilização em programas de controle do $\mathrm{CCU}^{3}$. A efetividade do exame e a longa fase detectável pré-clínica dessa neoplasia fazem com que o diagnóstico precoce, através do procedimento, seja a melhor estratégia para a prevenção ${ }^{4}$.

Informações sobre o conhecimento das mulheres em relação à finalidade do Papanicolaou e a sua

'Enfermeira. Especialista em Obstetrícia pela Escola Bahiana de Medicina e Saúde Pública. Bahia, Brasil. E-mail: tathy_1989@hotmail.com.

"Enfermeira. Mestre em Saúde Coletiva pela Universidade Estadual de Feira de Santana. Professora Assistente da Graduação em Enfermagem da Universidade do Estado da Bahia. Pesquisadora do Grupo de Pesquisa sobre o Cuidado em Enfermagem, Senhor do Bonfim. Bahia, Brasil. E-mail: magnaenf@yahoo.com.br. IIIEnfermeira. Mestre em Enfermagem pela Universidade Federal da Bahia. Professora Auxiliar da Graduação em Enfermagem da Universidade do Estado da Bahia. Pesquisadora do Grupo de Pesquisa sobre o Cuidado em Enfermagem. Bahia, Brasil. E-mail: gilvania.paixao@gmail.com. 
realização, são imprescindíveis para que as equipes de saúde conheçam a realidade local e utilizem estes dados para o planejamento e implementação de ações de saúde que objetivem a redução das atuais taxas de morbimortalidade por esse tipo de câncer ${ }^{5}$.

$\mathrm{O}$ estudo teve como objetivo descrever o conhecimento e a prática sobre o Papanicolaou das mulheres, entre 25 e 59 anos, residentes na área de abrangência da Unidade de Saúde da Família (USF) Nossa Senhora de Fátima do Alto da Maravilha, em Senhor do Bonfim, Bahia (BA).

A importância desta pesquisa consiste em produzir conhecimento para que equipes de saúde e gestores possam compreender as especificidades locais que envolvem a realização do Papanicolaou na população adscrita, e dessa maneira desenvolver adequado planejamento e implementação de ações para uma efetiva estruturação da prevenção do CCU.

\section{REVISÃO DE LITERATURA}

O CCU caracteriza-se pela replicação desordenada do epitélio de revestimento do útero, comprometendo o tecido subjacente (estroma), podendo invadir estruturas e órgãos contíguos ou à distância ${ }^{6}$. Este crescimento celular desordenado e consequente formação de tumores na cérvice uterina pode atingir mulheres jovens, mas ocorre principalmente naquelas com 35 anos ou mais?

principal fator associado à ocorrência dessa neoplasia é a infecção pelo Papilomavírus Humano (HPV), cuja principal via de transmissão é a sexual ${ }^{1,8}$. No entanto, para o desenvolvimento do CCU faz-se necessária, além da infecção pelo HPV, a existência de alguns co-fatores: tabagismo, precárias condições socioeconômicas, multiplicidade de parceiros sexuais, higiene íntima inadequada, alimentação pobre em vitamina $\mathrm{C}$, beta caroteno e folato, iniciação sexual precoce ${ }^{9}$.

O exame Papanicolaou (citologia oncótica) é prioritário para mulheres que já tenham iniciado a vida sexual, principalmente aquelas entre 25 a 59 anos de idade, e deve ser realizado uma vez por ano e, após dois exames anuais consecutivos negativos, a cada três anos ${ }^{1}$. Essa rotina não impossibilita a oferta do exame para as mulheres mais jovens ou mais velhas ${ }^{10}$. A finalidade do exame é a detecção das lesões precursoras para a instalação precoce da terapêutica sempre que necessário ${ }^{11}$.

A efetividade plena do rastreamento para redução da mortalidade por CCU ocorre apenas se os exames citopatológicos são conhecidos e aceitos pela população, além de serem repetidos em intervalos recomendados para a detecção da doença em estágios precoces ${ }^{12,13}$.

O número de óbitos por CCU no Brasil, em 2010, foi de 4.986 casos. Em relação às regiões brasileiras, a neoplasia cérvico-uterina apresenta elevada incidência, principalmente nas regiões Norte (24/100 mil) e
Centro-Oeste (28/100 mil), mas também apresenta indicadores significativos nas regiões Nordeste $(18 / 100$ mil), Sudeste $(15 / 100 \mathrm{mil})$ e Sul $(14 / 100 \mathrm{mil})^{2}$.

O baixo nível socioeconômico, as questões culturais associadas a problemas de acesso, mau funcionamento e precariedade dos serviços de saúde, como também o desconhecimento das mulheres sobre os benefícios da realização do Papanicolaou, explicam, em parte, porque cerca de 70,0\% dos casos de CCU são diagnosticados, no Brasil, em fase avançada, o que limita a possibilidade de cura, resultando nas elevadas taxas de morbimortalidade por essa neoplasia ${ }^{12}$.

\section{Metodologia}

Trata-se de um estudo quantitativo, descritivo de corte transversal. Foi realizado na área de abrangência da USF Nossa Senhora de Fátima, situada no bairro Alto da Maravilha no município de Senhor do Bonfim, BA. A USF abrange nove microáreas e possui um total de 1.281 famílias cadastradas ${ }^{14}$.

A população do estudo foi constituída por mulheres de 25 a 59 anos residentes na área de abrangência da USF. Por não existir na unidade de saúde uma relação de todas as mulheres de 25 a 59 anos para o sorteio da amostra, optou-se por sortear aleatoriamente uma microárea e, em seguida, sortear 50\% das famílias cadastradas nessa microárea, que possuíam mulheres entre 25 e 59 anos, para a partir daí obter a amostra para a realização do estudo.

Entre as nove microáreas que constituíam a área de abrangência da USF Nossa Senhora de Fátima, foi sorteada a nona microárea, sendo identificadas, através das fichas A (instrumento de cadastro das famílias) da respectiva microárea, 119 famílias cadastradas, das quais 82 possuíam mulheres de 25 a 59 anos. Foram sorteadas aleatoriamente 48 famílias, entre as 82 , equivalendo a aproximadamente $50 \%$ das famílias com mulheres na faixa etária alvo do estudo, resultando num total de 57 mulheres a serem entrevistadas, pois algumas famílias possuíam mais de uma mulher com idade para participar da pesquisa.

Os critérios de inclusão foram: mulheres integrantes das famílias cadastradas na nona microárea; com 25 a 59 anos; que iniciaram atividade sexual; não serem portadoras de distúrbios psíquicos que impedissem o fornecimento das respostas às questões propostas; não terem realizado histerectomia total ou parcial.

Os dados foram coletados durante a realização de visitas domiciliares, entre os meses de agosto e setembro de 2012, a partir da aplicação de um formulário semiestruturado, constituído por quatro blocos: dados sociodemográficos (idade, nível de escolaridade, raça/ cor, estado civil e renda mensal familiar); antecedentes gineco-obstétricos e sexualidade (idade na primeira relação sexual, número de parceiros desde o início da vida sexual, uso de método contraceptivo, história de 
doença sexualmente transmissível (DST), número de filhos, número de gestações e número de partos); conhecimento sobre o Papanicolaou (já ouviu falar sobre o exame, para que serve, conhecimento sobre a realização do exame na unidade de saúde do bairro); realização do exame (já fez o Papanicolaou alguma vez na vida, tempo de realização do último exame, frequência de realização, motivo para ter realizado o último exame e motivo para não ter realizado o citopatológico nos últimos três anos ou nunca ter se submetido ao procedimento).

Foram realizadas até três visitas às residências em que a cliente não se encontrava, mas mesmo com essa estratégia algumas não foram localizadas, tendo sido entrevistadas 47 mulheres.

As clientes que concordaram em participar, após terem sido informadas da importância e objetivo do estudo, assinaram o Termo de Consentimento Livre e Esclarecido, obedecendo aos aspectos éticos conforme determinação da Resolução no 466/2012, do Conselho Nacional de Saúde/Ministério da Saúde (MS), referente à pesquisa envolvendo seres humanos ${ }^{15}$.

Manteve-se preservada a identidade das entrevistadas, cada formulário foi identificado com uma numeração e a mesma foi utilizada para identificação das pesquisadas no banco de dados. $\mathrm{O}$ projeto foi aprovado pelo Comitê de Ética em pesquisa da Universidade do Estado da Bahia (parecer $n^{\circ} 57843$ ) em 12 de julho de 2012.

Em relação à classificação do conhecimento, utilizaram-se os seguintes critérios: conhecimento adequado quando a mulher já tinha ouvido falar do exame, e sabia que sua finalidade era detectar o câncer em geral ou, especificamente, o do colo uterino; e inadequado quando nunca tinha ouvido falar do exame, ou já tinha ouvido falar, mas não sabia que era utilizado para detectar câncer ou $\mathrm{CCU}^{16}$.

A avaliação da prática seguiu padrões estabelecidos pelo $\mathrm{MS}^{17}$, sendo considerada adequada quando o último exame realizado pela mulher tinha no máximo três anos, e inadequada quando a mulher havia feito o último exame há quatro anos ou mais ou nunca se submeteu ao procedimento.

Os dados coletados foram digitados no programa Statical Package for Social Sciences (SPSS) versão 9.0, e em seguida realizada a análise da digitação a partir das listagens das frequências simples das variáveis pesquisadas para a correção de possíveis erros. Para a análise do quesito raça/cor utilizou-se a resposta autorreferida.

Posteriormente, realizou-se análise descritiva da amostra estudada a partir das frequências simples das variáveis referentes aos aspectos sociodemográficos, antecedentes pessoais, antecedentes gineco-obstétricos e sexualidade, conhecimento sobre o exame Papanicolaou e realização do exame. Também foi efetuada análise bivariada para verificar as frequências das variáveis do estudo em relação ao conhecimento e à prática do exame.

\section{Resultados e Discussão}

De acordo com as características sociodemográficas das 47 entrevistadas, 20(42,6\%) apresentaram faixa etária entre 25 a 34 anos. Os dados relacionados à escolaridade mostraram que $18(38,3 \%)$ mulheres fizeram do $1^{\circ}$ ao $3^{30}$ ano do ensino médio, e $4(8,5 \%)$ não frequentaram a escola (não sabiam ler e escrever ou apenas assinavam o nome). Com relação à raça/cor da pele, $32(68,1 \%)$ autorreferiram ter cor parda; e quanto à situação conjugal, 15(31,9\%) eram solteiras. Entre as pesquisadas, 39(83,0\%) possuíam renda mensal familiar menor ou igual a um salário mínimo. Ver Tabela 1.

TABELA 1: Características sociodemográficas e antecedentes gineco-obstétricos das mulheres residentes na área de abrangência da USF Nossa Senhora de Fátima, Senhor do Bonfim, BA, 2012.

\begin{tabular}{|c|c|c|}
\hline Antecedentes & $f$ & $\%$ \\
\hline \multicolumn{3}{|l|}{ Idade (47) } \\
\hline 25 a 34 anos & 20 & 42,6 \\
\hline 35 a 44 anos & 12 & 25,5 \\
\hline 45 a 54 anos & 9 & 19,1 \\
\hline 55 anos ou mais & 6 & 12,8 \\
\hline \multicolumn{3}{|l|}{ Nível de Escolaridade (47) } \\
\hline Não alfabetizada & 4 & 8,5 \\
\hline Ensino Fundamental I & 8 & 17,0 \\
\hline Ensino Fundamental II & 13 & 27,7 \\
\hline Ensino Médio & 18 & 38,3 \\
\hline Ensino Superior & 4 & 8,5 \\
\hline \multicolumn{3}{|l|}{ Raça/cor de pele (47) } \\
\hline Preta & 8 & 17,0 \\
\hline Parda & 32 & 68,1 \\
\hline Branca & 4 & 8,5 \\
\hline Amarela/ Indígena & 3 & 6,4 \\
\hline \multicolumn{3}{|l|}{ Estado civil (47) } \\
\hline Solteira & 15 & 31,9 \\
\hline Casada & 14 & 29.8 \\
\hline Separada/divorciada/desquitada & 4 & 8,5 \\
\hline União estável & 11 & 23,4 \\
\hline Viúva & 3 & 6,4 \\
\hline \multicolumn{3}{|l|}{ Renda mensal familiar ${ }^{(*)}(47)$} \\
\hline Menor que1 SM & 39 & 83,0 \\
\hline Entre1 SM a 2 SM & 7 & 14,9 \\
\hline Maior ou igual que $3 \mathrm{SM}$ & 1 & 2,1 \\
\hline \multicolumn{3}{|l|}{ História de DST (47) } \\
\hline Sim & 1 & 2,1 \\
\hline Não & 45 & 95,8 \\
\hline Não sabe & 1 & 2,1 \\
\hline \multicolumn{3}{|l|}{ Tem filhos (47) } \\
\hline Sim & 43 & 91,5 \\
\hline Não & 4 & 8,5 \\
\hline \multicolumn{3}{|l|}{ Número de filhos (43) } \\
\hline 1 a 3 filhos & 37 & 86,0 \\
\hline 4 a 6 filhos & 3 & 7,0 \\
\hline 7 ou mais filhos & 3 & 7,0 \\
\hline \multicolumn{3}{|c|}{ Número de gestação (incluindo aborto) (47) } \\
\hline 0 a 3 gestações & 31 & 66,0 \\
\hline 4 ou mais gestações & 16 & 34,0 \\
\hline \multicolumn{3}{|l|}{ Número de partos (47) } \\
\hline 0 a 3 partos & 38 & 80,9 \\
\hline 4 ou mais partos & 9 & 19,1 \\
\hline
\end{tabular}


Em relação aos antecedentes gineco-obstétricos, apenas $1(2,1 \%)$ apresentou história de DST. Quanto a filhos, $43(91,5 \%)$ entrevistadas referiram ter filhos, sendo que $37(86,0 \%)$ possuíam de um a três filhos. De acordo com o número de gestações incluindo aborto, $31(66,0 \%)$ tiveram até três gestações, e 38(80,9\%) realizaram três partos ou menos, segunda a Tabela 1 .

Dados relacionados à sexualidade mostraram que $29(61,7 \%)$ entrevistadas iniciaram a atividade sexual com idade entre 12 e 17 anos. Com relação ao número de parceiros, desde o início da atividade sexual, 33(70,2\%) tiveram dois ou mais parceiros. $\mathrm{O}$ uso de algum método contraceptivo foi referido por $32(68,1 \%)$ mulheres, sendo que $18(56,3 \%)$ realizaram laqueadura e $8(25,0 \%)$ faziam uso de anticoncepcional oral combinado.

No que se refere ao conhecimento sobre o exame, todas as mulheres já ouviram falar sobre o Papanicolaou. Quando questionadas sobre a finalidade do citopatológico, 38(80,9\%) entrevistadas não detinham o conhecimento correto sobre o procedimento: $32(68,1 \%)$ responderam que o exame serve para prevenir doenças de modo geral, $5(10,7 \%)$ para tratar infecção vaginal e $1(2,1 \%)$ não soube, mostrando que parcela significativa das mulheres desconhecia a real finalidade do exame que é a prevenção do CCU. Contrastando com este achado, em pesquisa realizada em Juiz de Fora, MG, apenas 15\% das pesquisadas tinham conhecimento inadequado sobre o exame ${ }^{18}$, já no interior do Rio Grande do Norte, 53,9\% desconheciam a finalidade do Papanicolaou ${ }^{19}$.

O levantamento do conhecimento das mulheres sobre o exame é de grande relevância, pois constitui fator fundamental para avaliar as estratégias adotadas na prevenção do $\mathrm{CCU}^{20}$.

Entre as entrevistadas, 2(4,3\%) relataram não saber que o Papanicolaou era realizado na unidade de saúde do bairro. Desse modo, iniciativas devem ser tomadas quanto à prevenção do CCU, reforçando a informação e a orientação como forma de recrutar a população para a realização do exame ${ }^{21}$.

Quanto à prática do Papanicolaou, 42(89,4\%) entrevistadas realizaram o exame alguma vez na vida, conforme expõe a Tabela 2. Proporção semelhante foi encontrada em estudos realizados em São Paulo, SP, onde $86,1 \%$ das mulheres haviam realizado o citopatológico ${ }^{22}$, e em São Luís, MA, 82,4\% referiram ter se submetido ao procedimento pelo menos uma vez na vida ${ }^{23}$.

Observa-se que, na área de abrangência da USF Nossa Senhora de Fátima, há necessidade de esclarecimento e informações à população feminina a respeito do exame, uma vez que 5(10,6\%) mulheres nunca realizaram o procedimento, devendo essa população ser objeto de busca ativa e alvo de educação em saúde.

Destaca-se que 36(85,7\%) entrevistadas compareceram para a coleta do exame, nos últimos três anos, conforme a Tabela 2. O índice de prática adequada
TABELA 2: Prática do Papanicolaou pelas mulheres residentes na área de abrangência da USF Nossa Senhora de Fátima, Senhor do Bonfim, BA, 2012.

\begin{tabular}{|c|c|c|}
\hline Realização do exame & $f$ & $\%$ \\
\hline \multicolumn{3}{|l|}{$\begin{array}{l}\text { Fez o exame preventivo alguma vez na vida } \\
\text { (47) }\end{array}$} \\
\hline Sim & 42 & 89,4 \\
\hline Não & 5 & 10,6 \\
\hline \multicolumn{3}{|l|}{ Tempo de realização do último exame (42) } \\
\hline 3 anos ou menos & 36 & 85,7 \\
\hline 4 anos ou mais & 6 & 14,3 \\
\hline \multicolumn{3}{|l|}{ Frequência de realização do exame (42) } \\
\hline $\begin{array}{l}\text { Apenas quando aparece problema } \\
\text { Ginecológico }\end{array}$ & 1 & 2,4 \\
\hline De 6 em 6 meses & 3 & 7,1 \\
\hline Uma vez por ano & 22 & 52,4 \\
\hline De 2 em 2 anos & 4 & 9,5 \\
\hline De 3 em 3 anos & 1 & 2,4 \\
\hline Outro & 11 & 26,2 \\
\hline \multicolumn{3}{|l|}{ Motivo para realização do último exame (42) } \\
\hline Para prevenir o câncer de colo de útero & 2 & 4,8 \\
\hline Queixa ginecológica & 11 & 26,2 \\
\hline Recomendação do médico ou enfermeiro & 4 & 9,5 \\
\hline Recomendação de amigos ou parentes & 1 & 2,4 \\
\hline Por ser um exame de rotina & 10 & 23,8 \\
\hline Outro & 14 & 33,3 \\
\hline \multicolumn{3}{|l|}{$\begin{array}{l}\text { Motivo para a não realização do exame nos } \\
\text { últimos três anos ou para nunca ter feito (11) }\end{array}$} \\
\hline Ausência de problemas ginecológicos & 3 & 27,3 \\
\hline Falta de tempo & 1 & 9,1 \\
\hline Medo do resultado & 1 & 9,1 \\
\hline Outro & 6 & 54,5 \\
\hline
\end{tabular}

apresentado pelas mulheres deste estudo foi acima do encontrado na pesquisa realizada com mulheres argentinas $(30,5 \%)^{24}$.

Porém, o dado de cobertura deste estudo mostrase semelhante ao da pesquisa realizada com mulheres atendidas pela estratégia saúde da família do município de Feira de Santana, BA, $(87,4 \%)^{25}$, em Pelotas, RS, $(83,0 \%)^{26}$ e em pesquisa com mulheres atendidas em unidade básica de saúde (UBS) de Londrina, PR, $(80,0 \%)^{27}$.

$\mathrm{O}$ percentual de cobertura do exame de $\mathrm{Pa}$ panicolaou deste estudo foi positivo, visto que a Organização Mundial de Saúde considera um programa de prevenção do CCU de boa efetividade, quando a prática adequada do exame atinge $80,0 \%$ da população alvo, obtendo-se dessa maneira impacto significativo na redução da mortalidade por essa neoplasia ${ }^{17}$.

Ressalta-se que 22 (52,4\%) entrevistadas fizeram o exame anualmente e apenas $1(2,4 \%)$ o realizou no período preconizado pelo MS. A realização do $\mathrm{Pa}$ panicolaou em diferentes intervalos de tempo pode ser devido à falta de informações e de conhecimento dessas mulheres em relação à periodicidade recomendada pelo $\mathrm{MS}^{6}$.

A periodicidade inadequada da coleta para citopatológico pode ser reflexo da informação dada pelo profissional de saúde, como encontrado na pesquisa desen- 
volvida em Ribeirão Preto, SP, onde mulheres haviam recebido orientação sobre a importância da realização anual do exame ${ }^{28}$. Embora o profissional da unidade de saúde conheça a periodicidade preconizada pelo MS, a coleta anual ainda é orientada regularmente ${ }^{29}$.

Além disso, deve-se considerar que a realização anual do exame submete a mulher por maior número de vezes a um procedimento desconfortável e constrangedor, como também aumenta os custos do sistema de saúde por realizar desnecessariamente o procedimento, em intervalos de tempo menores que o preconizado pelo MS.

As principais causas citadas para a realização do último exame de Papanicolaou foram: 14(33,3\%) referiram outros motivos e entre estes o mais citado foi a prevenção de doenças em geral, 11 (26,2\%) mencionaram as queixas ginecológicas e $10(23,8 \%)$ por considerá-lo exame de rotina. Ver Tabela 2.

As justificativas relacionadas com a não realização do exame pelas entrevistadas nos últimos três anos ou para nunca ter feito foram variadas: $6(54,5 \%)$ referiram outros motivos e $3(27,3 \%)$ a ausência de queixas, condições estas que exercem grande influência na prática inadequada do Papanicolaou e colocam a saúde da mulher em risco.

Um dos motivos que levaram as mulheres a realizar o último Papanicolaou foi o aparecimento de sintomas, sendo característica da população de países em desenvolvimento a procura por serviços de saúde apenas quando apresentam alguma sintomatologia, devido ao entendimento de que não é necessário procurar um profissional de saúde quando não sente nada ${ }^{16}$.

Esse fato pode indicar a falta de conhecimento das entrevistadas sobre as ações preventivas, já que associam a realização do exame com a presença de alguma anormalidade. As mulheres devem ser informadas pelos profissionais de saúde a respeito das lesões precursoras do $\mathrm{CCU}$ e que estas lesões podem não apresentar sintomas, devendo o exame ser realizado com a finalidade da detecção precoce.

É importante que o serviço de saúde realize a busca ativa de mulheres para a realização do $\mathrm{Pa}$ panicolaou, e para isso é fundamental a participação do agente comunitário de saúde, por meio da visita domiciliar ${ }^{30}$. Nesse contexto, a sensibilização e a captação das mulheres serão mais efetivas e abrangentes se houver o envolvimento de toda a equipe da UBS.

Observou-se que a realização adequada do Papanicolaou neste estudo foi mais prevalente entre as mulheres com 40 a 59 anos de idade, pois $21(87,5 \%)$ entrevistadas, nessa faixa etária, se submeteram ao procedimento, segundo a Tabela 3. Tal achado corrobora estudo realizado em Campinas, SP, onde 83,3\% das mulheres com 40 anos ou mais mantinham prática adequada $^{31}$. No entanto, estes dados diferem do observado pelo MS, em que a maioria dos exames citopatológicos é realizada em mulheres com menos de 35 anos, devido a este grupo etário estar mais inserido nos serviços de saúde, em programas relacionados à fase reprodutiva ${ }^{12}$.

Observou-se que mesmo entre as entrevistadas com baixa escolaridade (não alfabetizadas ou que cursaram o Ensino Fundamental I), a prática do exame foi adequada, pois $11(91,7 \%)$ mulheres realizaram o exame nos últimos três anos, conforme a Tabela 3. Achado semelhante foi encontrado em pesquisa realizada em Ribeirão Preto, SP, onde as mulheres que possuíam o $1^{\underline{O}}$ grau e as analfabetas apresentaram uma maior adesão ao Papanicoloau ${ }^{30}$.

Em contrapartida, a literatura relata que quanto menor o nível de escolaridade maior é a prevalência de mulheres sem cobertura pelo exame de detecção precoce do $\mathrm{CCU}^{22}$. Pesquisa realizada, em Feira de Santana, $\mathrm{BA}$, mostra que entre as mulheres que não aderiram ao exame, $38,5 \%$ nunca tinham frequentado a escola, evidenciando elevada prevalência de não adesão entre as mulheres com baixa escolaridade, sendo encontrada associação estatisticamente significante ${ }^{25}$.

Em relação à raça/cor de pele, destacaram-se as $11(27,5 \%)$ mulheres que se autorreferiram preta/parda e possuíam prática inadequada do exame, conforme a Tabela 3. Estudo desenvolvido, no município de Rio Grande, RS, evidenciou que as maiores razões de prevalência para a não realização do exame ocorreram entre as mulheres de cor preta ou parda $(70,9 \%)^{32}$. Este fato se agrava ao considerar que o risco de mulheres negras contraírem o CCU é duas vezes mais frequente se comparado com as mulheres brancas ${ }^{17}$.

Todas as entrevistadas que se autorreferiram brancas ou amarelas/indígenas realizaram o exame adequadamente, evidenciando a existência de desigualdade de raça/cor quanto ao acesso ao exame de prevenção da neoplasia, sendo essa iniquidade um importante problema a ser trabalhado pelos gestores e profissionais de saúde.

Ao analisar a situação conjugal, constatou-se que, entre as mulheres sem companheiro, $6(27,3 \%)$ não possuíam prática adequada do exame e 5 (20,0\%) mulheres casadas ou em união estável mencionaram esse mesmo comportamento. Em estudo realizado no Rio Grande, RS, 72,6\% das mulheres solteiras apresentaram maior probabilidade de realização inadequada do exame ${ }^{32}$. Em relação ao número de parceiros, desde o início da vida sexual, 9 (27,3\%) mulheres com mais de dois parceiros sexuais revelaram realização inadequada do Papanicolaou de acordo com a Tabela 3.

O início precoce da vida sexual e o número elevado de parceiros podem ser considerados fatores de risco para a ocorrência do CCU, pois as mulheres ficam mais expostas à infecção pelo HPV, contribuindo para o desenvolvimento da neoplasia ${ }^{33}$.

Entre as entrevistadas que utilizam algum método contraceptivo, ressalta-se que nenhuma referiu 
TABELA 3: Distribuição das frequências de realização (prática) do exame Papanicolaou segundo características sociodemográficas e outras variáveis USF Nossa Senhora de Fátima, Senhor do Bonfim, BA, 2012. (N=47)

\begin{tabular}{|c|c|c|c|c|}
\hline \multirow{3}{*}{$\begin{array}{c}\text { Características sociodemográficas, antecedentes gineco- } \\
\text { obstétricos e sexualidade }\end{array}$} & \multicolumn{4}{|c|}{ Prática do exame preventivo } \\
\hline & \multicolumn{2}{|c|}{ Prática adequada } & \multicolumn{2}{|c|}{ Prática inadequada } \\
\hline & $f$ & $\%$ & $f$ & $\%$ \\
\hline \multicolumn{5}{|l|}{ Idade } \\
\hline 25 a 39 anos & 15 & 65,2 & 8 & 34,8 \\
\hline 40 a 59 anos & 21 & 87,5 & 3 & 12,5 \\
\hline \multicolumn{5}{|l|}{ Nível de Escolaridade } \\
\hline Não alfabetizada/ Ensino & 11 & 91,7 & 1 & 8,3 \\
\hline \multicolumn{5}{|l|}{ Fundamental I } \\
\hline Ensino Fundamental II / Ensino & 22 & 71,0 & 9 & 29,0 \\
\hline \multicolumn{5}{|l|}{ Médio } \\
\hline Ensino Superior & 3 & 75,0 & 1 & 25,0 \\
\hline \multicolumn{5}{|l|}{ Raça/cor de pele } \\
\hline Preta / Parda & 29 & 72,5 & 11 & 27,5 \\
\hline Branca & 4 & 100 & 0 & 0,0 \\
\hline Amarela / Indígena & 3 & 100 & 0 & 0,0 \\
\hline \multicolumn{5}{|l|}{ Estado civil } \\
\hline Solteira/ Separada/divorciada/desquitada / Viúva & 16 & 72,7 & 6 & 27,3 \\
\hline Casada / União estável & 20 & 80,0 & 5 & 20,0 \\
\hline \multicolumn{5}{|l|}{ Número de parceiros sexuais desde o início da vida sexual } \\
\hline 1 parceiro & 12 & 85,7 & 2 & 14,3 \\
\hline 2 parceiros ou mais & 24 & 72,7 & 9 & 27,3 \\
\hline \multicolumn{5}{|l|}{ Uso de método contraceptivo } \\
\hline Sim & 24 & 75,0 & 8 & 25,0 \\
\hline Não & 12 & 80,0 & 3 & 20,0 \\
\hline \multicolumn{5}{|l|}{ Tem filhos } \\
\hline Sim & 33 & 76,7 & 10 & 23,3 \\
\hline Não & 3 & 75,0 & 1 & 25,0 \\
\hline \multicolumn{5}{|l|}{ Número de gestação (incluindo aborto) } \\
\hline 0 a 3 gestações & 24 & 77,4 & 7 & 22,6 \\
\hline 4 ou mais gestações & 12 & 75,0 & 4 & 25,0 \\
\hline \multicolumn{5}{|l|}{ Número de partos } \\
\hline 0 a 3 partos & 30 & 78,9 & 8 & 21,1 \\
\hline 4 ou mais partos & 6 & 66,7 & 3 & 33,3 \\
\hline
\end{tabular}

o uso de preservativos, $8(25,0 \%)$ não fizeram o citopatológico de forma correta. Ver Tabela 3. Segundo o MS, os anticoncepcionais são usados principalmente por mulheres com vida sexual ativa, que não fazem o uso adequado de preservativo, aumentando por consequência o risco de contrair o HPV ${ }^{12}$.

Entre as entrevistadas que referiram ter filhos, $10(23,3 \%)$ não realizaram o Papanicolaou adequadamente, conforme a Tabela 3.

Pesquisa realizada em Puerto Leoni, Argentina, mostrou proporções elevadas de prática inadequada em mulheres com maior paridade, assim o elevado número de filhos apresentou-se como um obstáculo para a realização do exame nos últimos três anos ${ }^{24}$. A quantidade de filhos influencia no autocuidado da mulher em relação a sua saúde, pois os afazeres domésticos e o cotidiano de uma mãe fazem com que ela deixe de cuidar de sua saúde, se dedicando mais à família, contribuindo para o aumento da detecção tardia do $\mathrm{CCU}^{34}$.

\section{CONClusão}

Os resultados do estudo mostram que a maior parte das mulheres possuía prática adequada em relação ao Papanicolaou, ou seja, se submeteram ao exame nos três anos anteriores à pesquisa. No entanto, prevaleceu o desconhecimento sobre a finalidade da coleta para o exame citopatológico.

A prática inadequada foi observada em maior proporção nas mulheres com idade entre 25 a 39 anos, pretas/pardas, que vivem sem companheiro, possuem multiplicidade de parceiros sexuais, fazem uso de algum método contraceptivo sem referir o preservativo, multigestas e multíparas.

Embora a prática do Papanicolaou esteja de acordo com o protocolo do MS, ainda existem mulheres que realizam o exame irregularmente ou nunca se submeteram a ele e que não estão orientadas quanto a sua periodicidade. 
Uma limitação importante do estudo de corte transversal consiste no viés de memória, pois nem todas as mulheres possuem recordações fidedignas dos fatos passados, podendo haver equívocos ao relatar situações ocorridas em anos anteriores.

Conhecer a realidade de uma determinada população sobre aspectos que envolvem a prevenção do CCU é o primeiro passo para definir estratégias de intervenções mais eficientes às reais necessidades da comunidade, contribuindo, assim, para a redução dos indicadores de morbimortalidade por este tipo de câncer, impactando de maneira positiva na qualidade de vida das mulheres.

\section{REFERÊNCIAS}

1.Ministério da Saúde (Br). Instituto Nacional de Câncer (INCA). Estimativa 2010: incidência de câncer no Brasil / Instituto Nacional de Câncer. Rio de Janeiro: Ministério da Saúde; 2009.

2.Ministério da Saúde (Br). Instituto Nacional de Câncer (INCA). Estimativa 2012: incidência de câncer no Brasil. Rio de Janeiro: Ministério da Saúde; 2011.

3.Ministério da Saúde (Br). Instituto Nacional do Câncer (INCA). Programa Nacional de Controle do Câncer do Colo do Útero. Rio de Janeiro: Ministério da Saúde; 2010. 4.Medeiros VCRD, Medeiros RC, Moraes LM, Menezes FJB, Ramos ESN, Saturnino ACRD.

Câncer de colo de útero: análise epidemiológica e citopatológica no Estado do Rio Grande do Norte. Rev bras anal clin. 2005; 37: 227-31.

5.Souza AB, Borba PC. Exame citológico e os fatores determinantes na adesão de mulheres na estratégia saúde da família do município de Assaré. Cad cult ciênc. 2008; 2(1): 36-45.

6.Instituto Nacional de Câncer. Plano de ação para redução da incidência e mortalidade por câncer do colo do útero: sumário executivo. Programa Nacional de Controle do Câncer do Colo do Útero. Rio de Janeiro: Ministério da Saúde; 2010.

7.Hailu M, Mariam DH. Patient side cost and its predictors for cervical cancer in Ethiopia: a cross sectional hospital based study. BMC Cancer. 2013; [cited in $2014 \mathrm{Feb}$ 14] 13(69): 1-8. Available in: http://www.ncbi.nlm.nih. gov/pmc/articles/PMC3576296/?tool=pubmedhttp:// dx.doi.org/10.1186/1471-2407-13-69.

8.Saslow D, Solomon D, Lawson W H, Killackey M, Kulasingam S, Cain J, et al. American Cancer Society, American Society for Colposcopy and Cervical Pathology, and American Society for Clinical Pathology Screening Guidelines for the Prevention and Early Detection of Cervical Cancer. CA Cancer J Clin. 2012; [cited in 2014 Fev 14] 62 (3): 147-72. Available in: http://onlinelibrary. wiley.com/doi/10.3322/caac.21139/pdf.

9.Ministério da Saúde (Br). Instituto Nacional de Câncer (INCA). Secretaria de Atenção à Saúde. Coordenação de Prevenção e Vigilância de Câncer. Estimativas 2008: Incidência de câncer no Brasil. Rio de Janeiro: Ministério da Saúde; 2007.
10.Pinho AA, Mattos MCFI. Validade da citologia cervicovaginal na detecção de lesões pré-neoplásicas e neoplásicas de colo de útero. J bras pat med lab. 2002; 38: 225-31.

11.Nascimento LC, Nery IS, Silva AO. Conhecimento cotidiano de mulheres sobre a prevenção do câncer de colo do útero. Rev enferm UERJ. 2012; [citado em 14 fev 2014] 20: 476-80. Disponível em: http://www.facenf. uerj.br/v20n4/v20n4a11.pdf.

12.Instituto Nacional de Câncer. Coordenação de Prevenção e Vigilância. Falando sobre câncer do colo do útero. Rio de Janeiro: Ministério da Saúde; 2002.

13.Weiland DL, Reimers LL, Wu E, Nathan LM, Gruenberg T, Abadi M, et al. Performance of implementing Guideline Driven Cervical Cancer Screening Measures in an Inner City Hospital System. J Low Genit Tract Dis. 2011; [cited in 2014 Feb 14] 15: 296-302. Available in: http://www.ncbi.nlm.nih.gov/pmc/articles/ PMC3681613/pdf/nihms467127.pdf.

14.Ministério da Saúde (Br). Sistema de Informação da Atenção Básica (SIAB). Consolidado das famílias cadastradas do ano de 2011. Município Senhor do Bonfim, BA, Zona Urbana, PSF Nossa Senhora de Fátima. Brasília (DF): SIAB; 2012.

15.Ministério da Saúde (Br). Conselho Nacional de Saúde. Resolução n 466 de 12 de dezembro de 2012: Diretrizes e normas regulamentadoras de pesquisa em seres humanos. [citado em 14 fev 2014] Disponível em: http:// conselho.saude.gov.br/resolucoes/2012/Reso466.pdf. 16.Brenna SMF, Hardy EE, Zeferino LC, Namura I. Conhecimento, atitude e prática do exame de Papanicolaou em mulheres com câncer de colo uterino. Cad Saúde Pública. 2001; 17: 909-14.

17.Ministério da Saúde (Br). Controle dos cânceres do colo do útero e da mama. Secretaria de Atenção à Saúde, Departamento de Atenção Básica. Brasilia (DF): Editora MS; 2006. 18.Afonso VW, Ribeiro LC, Bertocchi FM, Diniz ALL, Passos CS, Paula EA. Conhecimentos, atitudes e práticas acerca de exames ginecológicos preventivos por trabalhadoras do terceiro setor. HU rev. 2011; 37: 431-9.

19.Fernandes JV, Rodrigues SHL, Costa YGAS, Silva LCM, Brito AML, Azevedo JWV, Nascimento ED, Azevedo PRM, Fernandes TAAM. Conhecimentos, atitudes e prática do exame de Papanicolaou por mulheres, Nordeste do Brasil. Rev Saude Publica. 2009; 43: 851-8. 20.Moura ADA, Silva SMG, Farias LM, Feitoza AR. Conhecimento e motivações das mulheres acerca do exame de papanicolaou: subsídios para a prática de enfermagem. Rev RENE. 2010; 11: 94-104.

21.Gonçales MB, Barbien M, Gabrielloni MC. Teste de Papanicolaou: construção e validação de material educativo para usuárias de serviços de saúde. Ciên saúde coletiva. 2008; 5(20): 39-44.

22.Pinho AA, França JI, Schraiber LB, D'Oliveira AFPL. Cobertura e motivos para a realização ou não do teste de papanicolaou no município de São Paulo. Cad Saúde Pública. 2003; 19: 303-13.

23. Oliveira MMHN, Silva AAM, Brito LMO, Coimbra LC. Cobertura e fatores associados à não realização do exame preventivo de papanicolaou em São Luís, Maranhão. Rev bras epidemiol. 2006; 9: 325-34. 
24.Gamarra CJ, Paz EPA, Griep RH. Conhecimentos, atitudes e prática do exame de Papanicolaou entre mulheres argentinas. Rev Saude Publica. 2005; 39: 270-6.

25.Andrade MS, Almeida MMG, Araújo TM, Santos KOB. Adesão ao Papanicolaou entre mulheres atendidas pela estratégia de saúde da família em Feira de Santana - BA [dissertação de mestrado]. Feira de Santana (BA): Universidade Estadual de Feira de Santana; 2010.

26. Hackenhaar AA, Cesar JA, Domingues MR. Exame citopatológico de colo uterino em mulheres com idade entre 20 e 59 anos em Pelotas, RS: prevalência, foco e fatores associados à sua não realização. Rev bras epidemiol 2006; 9: 103 - 11.

27.Silva DW, Andrade SM, Soares DA, Turini B, Schneck CA, Lopes MLS. Cobertura e fatores associados com a realização do exame Papanicolaou em município do Sul do Brasil. Rev bras ginecol obstet. 2006; 28(1): 24-31. 28. Oliveira MM. A Prevenção do câncer do colo do útero, no contexto da estratégia saúde da família, da área básica da distrital-oeste/sumarezinho, do município de Ribeirão Preto-SP [dissertação de mestrado]. Ribeirão Preto (SP): Universidade de São Paulo; 2003.

29.Pinho MCV. Avaliação do programa de controle do câncer do colo do útero e de mama em Londrina [dissertação de mestrado]. Maringá (PR): Universidade
Estadual de Maringá; 2005.

30.Ramos AS, Palha PF, Costa JML, Sant'Anna SC, Lenza NFB. Perfil de mulheres de 40 a 49 anos cadastradas em um núcleo de saúde da família, quanto à realização do exame preventivo de papanicolaou. Rev Latino-Am Enfermagem. 2006; 14: 170-4.

31.Amorim VMSL. As práticas preventivas para o câncer de mama e do colo do útero por mulheres de 40 anos ou mais de idade no município de Campinas - SP [dissertação de mestrado]. Campinas (SP): Universidade Estadual de Campinas; 2005.

32.Cesar JA, Horta BL, Gomes G, Houlthausen RS, Kaercher A, Iastrenski FM. Fatores associados à não realização de exame citopatológico de colo uterino no extremo Sul do Brasil. Cad Saúde Pública. 2003; 19: 1365-72.

33.Chubaci RYS, Merighi MAB. Exame para detecção precoce do câncer cérvico-uterino: vivência de mulheres das cidades de Kobe e Kawasaki, Japão e São Paulo, Brasil. Rev Bras Saúde Mater Infant. 2005; 5: 471-81.

34.Maciel I, Kunz JZ. Assistência de enfermagem à mulher na promoção e prevenção do câncer do colo uterino e mama: fundamentado na Teoria de Dorothea Elizabeth Orem [trabalho de conclusão de graduação]. Chapecó (SC): Universidade Comunitária da Região de Chapecó; 2010. 